\title{
Supplement to: Testing for instability in covariance structures
}

\author{
Chihwa Kao* \\ Lorenzo Trapani ${ }^{\dagger}$ \\ Syracuse University \\ Cass Business School \\ Giovanni Urga ${ }^{\ddagger}$ \\ Cass Business School and Università di Bergamo
}

August 2, 2016

This supplement contains some further material for the paper "Testing for instability in covariance structures".

In particular, in this note we consider: the extension of results derived in the main paper to residuals (Section 1); a related empirical exercise, where we study the stability of the covariance matrix of the error term of a VAR model for leading currency exchange rates (Section 2); a data appendix, where we describe the data employed in both the application in the main paper and the one in Section 2 (Section 3); the statements and proofs of several lemmas, either used in the main paper or in this supplement, and of the results derived in here (Section 4); all the tables that refer to simulations in the main paper, the critical values employed for the empirical applications in this supplement and in the main paper, and the empirical exercise in this supplement (Section 5).

\section{Applications to the residuals of a multivariate regression}

In this section, we extend the results developed in the main paper by proposing a test for the time stability of the covariance matrix (and its eigensystem) of the error term in a multivariate regression, such as a VAR.

Consider the following multivariate regression model

$$
y_{t}=\beta x_{t}+\varepsilon_{t},
$$

where $t=1, \ldots, T$ and $y_{t}$ and $\varepsilon_{t}$ are $n \times 1$ vectors, $x_{t}$ is of dimension $q \times 1$ and the matrix of

\footnotetext{
${ }^{*}$ Center for Policy Research, 426 Eggers Hall, Syracuse University, Syracuse, NY 13244-1020 (USA). Tel. +.1. 315.4433233, Fax. +1-315-443-1081, e-mail: dkao@maxwell.syr.edu

${ }^{\dagger}$ Centre for Econometric Analysis, Faculty of Finance, Cass Business School, 106 Bunhill Row, London EC1Y 8TZ (U.K.). Tel. +.44.20.70405260 Fax. +.44.20.70408881, e-mail: L.Trapani@city.ac.uk

${ }^{\ddagger}$ Centre for Econometric Analysis, Faculty of Finance, Cass Business School, 106 Bunhill Row, London EC1Y 8TZ (U.K.) and Bergamo University (Italy). Tel. +.44.20.70408698 Fax. +.44.20.70408881, e-mail: g.urga@city.ac.uk
} 
regressors $\beta$ has dimensions $n \times q$. Of course, $n$ can be equal to 1 , thereby recovering the usual univariate regression; alternatively, the regressors $x_{t}$ can be the lags of $y_{t}$, thus having a VAR. Bai (2000) considers a test for structural changes in a VAR, extending it to test for changes in the covariance matrix of the error term. The theory developed in this section also extends naturally to the case of linear or polynomial trends among the regressors - see also Aue et al. (2012).

Let $\Sigma_{\varepsilon}=E\left(\varepsilon_{t} \varepsilon_{t}^{\prime}\right)$ be the covariance matrix of the error term $\varepsilon_{t}$; using the same notation as above, we denote its eigenvalues and eigenvectors as $\lambda_{i}^{\varepsilon}$ and $x_{i}^{\varepsilon}$ respectively, satisfying the relationship $\Sigma_{\varepsilon} x_{i}^{\varepsilon}=\lambda_{i}^{\varepsilon} x_{i}^{\varepsilon}$ for $i=1, \ldots, n$.

Tests for the time stability of $\Sigma_{\varepsilon}$ and of its eigensystem are based on the residuals $\hat{\varepsilon}_{t}=$ $y_{t}-\hat{\beta} x_{t}$, where $\hat{\beta}=\left[\sum_{t=1}^{T} y_{t} x_{t}^{\prime}\right]\left[\sum_{t=1}^{T} x_{t} x_{t}^{\prime}\right]^{-1}$. Hence, the full sample estimator of $\Sigma_{\varepsilon}$ is defined as $\hat{\Sigma}_{\varepsilon}=T^{-1} \sum_{t=1}^{T} \hat{\varepsilon}_{t} \hat{\varepsilon}_{t}^{\prime}$. As above, for a given point in time $\lfloor T \tau\rfloor$ with $\tau \in[0,1]$, we use the subscript $\tau$ to denote quantities calculated using the subsamples $t=1, \ldots,\lfloor T \tau\rfloor$. In particular, we extensively use the sequence of partial sample estimators $\hat{\Sigma}_{\varepsilon, \tau}=(T \tau)^{-1} \sum_{t=1}^{\lfloor T \tau\rfloor} \hat{\varepsilon}_{t} \hat{\varepsilon}_{t}^{\prime}$. We also employ the following definitions: $w_{t}^{\varepsilon}=\operatorname{vec}\left(\varepsilon_{t} \varepsilon_{t}^{\prime}\right)$ and $\bar{w}_{t}^{\varepsilon}=\operatorname{vec}\left(\varepsilon_{t} \varepsilon_{t}^{\prime}-\Sigma_{\varepsilon}\right)$.

We need the following Assumptions, which are closely related to Assumptions 1-3 in the main paper.

Assumption R1. (i) Assumptions 1(i)-1(ii) hold for $x_{t}$; (ii) Assumptions 1(i)-1(ii) hold for $\varepsilon_{t}$; (iii) let $w_{t}^{x \varepsilon}=\operatorname{vec}\left(\varepsilon_{t} x_{t}^{\prime}\right)$; it holds that: (a) $E\left(w_{t}^{x \varepsilon}\right)=0$ for all $t$; (b) defining $V_{T}^{x \varepsilon}$

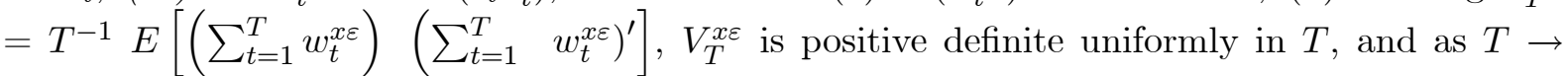
$\infty, V_{T}^{x \varepsilon} \rightarrow V^{x \varepsilon}$ with $\left\|V^{x \varepsilon}\right\|<\infty$; (c) letting $w_{i t}^{x \varepsilon}$ be the $i$-th element of $w_{t}^{x \varepsilon}$ and defining $S_{i T, m}^{x \varepsilon} \equiv \sum_{t=m+1}^{m+T} w_{i t}^{x \varepsilon}$, there exists a positive definite matrix $\bar{\Omega}^{x \varepsilon}=\left\{\varpi_{i j}^{x \varepsilon}\right\}$ such that $T^{-1}\left|E\left[S_{i T, m}^{x \varepsilon} S_{j T, m}^{x \varepsilon}\right]-\varpi_{i j}^{x \varepsilon}\right| \leq M T^{-\psi}$, for all $i$ and $j$ and uniformly in $m$, with $M$ a constant and $\psi>0$; (iv) (a) letting $V_{T}^{\varepsilon}=T^{-1} E\left[\left(\sum_{t=1}^{T} \bar{w}_{t}^{\varepsilon}\right)\left(\sum_{t=1}^{T} \bar{w}_{t}^{\varepsilon}\right)^{\prime}\right], V_{T}^{\varepsilon}$ is positive definite uniformly in $T$, and as $T \rightarrow \infty, V_{T}^{\varepsilon} \rightarrow V^{\varepsilon}$ with $\left\|V^{\varepsilon}\right\|<\infty$; (b) letting $\bar{w}_{i t}^{\varepsilon}$ be the $i$-th element of $\bar{w}_{t}^{\varepsilon}$ and defining $S_{i T, m}^{\varepsilon} \equiv \sum_{t=m+1}^{m+T} \bar{w}_{i t}^{\varepsilon}$, there exists a positive definite matrix $\bar{\Omega}^{\varepsilon}=\left\{\varpi_{i j}^{\varepsilon}\right\}$ such that $T^{-1}\left|E\left[S_{i T, m}^{\varepsilon} S_{j T, m}^{\varepsilon}\right]-\varpi_{i j}^{\varepsilon}\right| \leq M^{\prime} T^{-\psi^{\prime}}$, for all $i$ and $j$ and uniformly in $m$, with $\psi^{\prime}>0$.

Assumption R2. Let $\Psi_{l}^{\varepsilon}=E\left(\bar{w}_{t}^{\varepsilon} \bar{w}_{t-l}^{\varepsilon \prime}\right)$; it holds that (i) $\sum_{l=0}^{\infty} l^{s}\left\|\Psi_{l}^{\varepsilon}\right\|<\infty$ for some $s \geq 1$; (ii) $\sup _{t} E\left\|\varepsilon_{t}\right\|^{4 r}<\infty$ for some $r>2$; (iii) letting $\Omega_{T}^{l \varepsilon}=T^{-1} E\left\{\sum_{t=1}^{T} \operatorname{vec}\left[\bar{w}_{t}^{\varepsilon} \bar{w}_{t-l}^{\varepsilon \prime}-E\left(\bar{w}_{t}^{\varepsilon} \bar{w}_{t-l}^{\varepsilon \prime}\right)\right]\right.$ $\left.\operatorname{vec}\left[\bar{w}_{t}^{\varepsilon} \bar{w}_{t-l}^{\varepsilon \prime}-E\left(\bar{w}_{t}^{\varepsilon} \bar{w}_{t-l}^{\varepsilon \prime}\right)\right]^{\prime}\right\}, \Omega_{T}^{l \varepsilon}$ is positive definite uniformly in $T$, and $\Omega_{T}^{l \varepsilon} \rightarrow \Omega^{l \varepsilon}$ with $\left\|\Omega^{l \varepsilon}\right\|<\infty$ for every $l$.

Assumption R3. Assumption 3 in the main paper holds for the matrix $\Sigma_{\varepsilon}$.

Let $\hat{w}_{t}^{\varepsilon}=\operatorname{vec}\left(\hat{\varepsilon}_{t} \hat{\varepsilon}_{t}^{\prime}\right)$. In order to estimate the long run covariance matrix $V^{\varepsilon}$ defined in Assumption R1(iv)(b), we propose the weighted sum-of-covariance estimator $\tilde{V}_{\tau}^{\varepsilon}=\left(\hat{\Psi}_{0,\lfloor T \tau\rfloor}^{\varepsilon}+\hat{\Psi}_{0,1-\lfloor T \tau\rfloor}^{\varepsilon}\right)$ 
$+\sum_{l=1}^{m}\left(1-\frac{l}{m}\right)\left[\left(\hat{\Psi}_{l,\lfloor T \tau\rfloor}^{\varepsilon}+\hat{\Psi}_{l,\lfloor T \tau\rfloor}^{\varepsilon \prime}\right)+\left(\hat{\Psi}_{l, 1-\lfloor T \tau\rfloor}^{\varepsilon}+\hat{\Psi}_{l, 1-\lfloor T \tau\rfloor}^{\varepsilon \prime}\right)\right]$, where $\hat{\Psi}_{l,\lfloor T \tau\rfloor}^{\varepsilon}=\frac{1}{T-(l+1)} \sum_{t=l+1}^{\lfloor T \tau\rfloor}$ $\left[\hat{w}_{t}^{\varepsilon}-\operatorname{vec}\left(\hat{\Sigma}_{\varepsilon}\right)\right]\left[\hat{w}_{t-l}^{\varepsilon}-\operatorname{vec}\left(\hat{\Sigma}_{\varepsilon}\right)\right]^{\prime}$, and similarly $\hat{\Psi}_{l, 1-\lfloor T \tau\rfloor}^{\varepsilon}$.

In order to set up the test statistic, consider the following notation. When estimating the eigensystem of $\Sigma_{\varepsilon}$, we define the estimators of $\left(\lambda_{i}^{\varepsilon}, x_{i}^{\varepsilon}\right)$ as the solution to $\hat{\Sigma}_{\varepsilon} \hat{x}_{i}^{\varepsilon}=\hat{\lambda}_{i}^{\varepsilon} \hat{x}_{i}^{\varepsilon}$, where $\hat{\lambda}_{i}^{\varepsilon}$ and $\hat{x}_{i}^{\varepsilon}$ denote the estimates of $\lambda_{i}^{\varepsilon}$ and $x_{i}^{\varepsilon}$ respectively; the Pearson-Hotelling estimator of the eigenvectors is defined as $\hat{\gamma}_{i}^{\varepsilon}=\sqrt{\hat{\lambda}_{i}^{\varepsilon}} \hat{x}_{i}^{\varepsilon}$. Similarly, the partial sample estimators of the eigenvalues and eigenvectors are the solutions to $\hat{\Sigma}_{\varepsilon, \tau} \hat{x}_{i, \tau}^{\varepsilon}=\hat{\lambda}_{i, \tau}^{\varepsilon} \hat{x}_{i, \tau}^{\varepsilon}$, and we define $\hat{\gamma}_{i, \tau}^{\varepsilon}=\sqrt{\hat{\lambda}_{i, \tau}^{\varepsilon}} \hat{x}_{i, \tau}^{\varepsilon}$. Thence, let $\lambda^{\varepsilon} \equiv\left[\lambda_{1}^{\varepsilon}, \ldots, \lambda_{n}^{\varepsilon}\right]^{\prime}$ be the $n$-dimensional vector containing the eigenvalues sorted in descending order; $\Gamma^{\varepsilon} \equiv\left[\gamma_{1}^{\varepsilon}, \ldots, \gamma_{n}^{\varepsilon}\right]$; and let $D_{\lambda \gamma}^{\varepsilon} \equiv\left[x_{1}^{\varepsilon} \otimes x_{1}^{\varepsilon}, \ldots, x_{n}^{\varepsilon} \otimes x_{n}^{\varepsilon}, v_{\gamma, 1}^{\varepsilon \prime}, \ldots, v_{\gamma, n}^{\varepsilon \prime}\right]^{\prime}$, with $v_{\gamma, i}^{\varepsilon}=\frac{1}{2} \frac{x_{i}^{\varepsilon}}{\sqrt{\lambda_{i}^{\varepsilon}}}$ $\left(x_{i}^{\varepsilon \prime} \otimes x_{i}^{\varepsilon \prime}\right)+\sum_{k \neq i} \frac{\sqrt{\lambda_{i}^{\varepsilon}} x_{k}^{\varepsilon}}{\lambda_{i}^{\varepsilon} \lambda_{k}^{\varepsilon}}\left(x_{i}^{\varepsilon \prime} \otimes x_{k}^{\varepsilon \prime}\right)$. Estimation of $D_{\lambda \gamma}^{\varepsilon}$ is based on $\hat{\lambda}_{i}^{\varepsilon}$ and $\hat{x}_{i}^{\varepsilon}$.

Based on the notation spelt out above, we define the test statistic for the time stability of $\Sigma_{\varepsilon}$ and its eigensystem. Let

$$
\tilde{S}^{\varepsilon}(\tau)=R \times D_{\lambda \gamma}^{\varepsilon} \times\left[S^{\varepsilon}(\tau)-\frac{\lfloor T \tau\rfloor}{T} S^{\varepsilon}(1)\right],
$$

where $S^{\varepsilon}(\tau)$ is the CUSUM process defined as $\sum_{t=1}^{\lfloor T \tau\rfloor} \operatorname{vec}\left(\hat{\varepsilon}_{t} \hat{\varepsilon}_{t}^{\prime}\right), \tilde{S}^{\varepsilon}(\tau)=0$ for $\tau \leq \frac{1}{T}$ or $\geq 1-\frac{1}{T}$, and $R$ is defined in equation (13) in the main paper. The test statistic is defined as

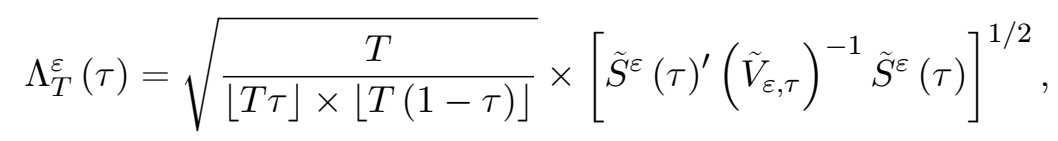

with $\tilde{V}_{\varepsilon, \tau}=R D_{\lambda \gamma}^{\varepsilon} \tilde{V}_{\tau}^{\varepsilon} D_{\lambda \gamma}^{\varepsilon \prime} R^{\prime}$

Let $\Lambda_{T}^{\varepsilon *} \equiv \sup _{n q \leq\lfloor T \tau\rfloor \leq T-n q} \Lambda_{T}^{\varepsilon}(\tau)$. The following theorem summarizes the asymptotics of $\Lambda_{T}^{\varepsilon *}$ under the null and under the alternative. Similarly to Theorem 4 in the main paper, we define the critical value $c_{\alpha, T}$ to be such that, under $H_{0}, P\left[\Lambda_{T}^{\varepsilon *} \leq c_{\alpha, T}\right]=1-\alpha$ for some $\alpha \in[0,1]$.

Theorem 1 Let Assumptions R1-R3 hold, and let $p$ be defined as in Theorem 3 in the main paper. Under the null, as $(m, T) \rightarrow \infty$ with $\frac{\sqrt{\ln \ln T}}{m}+\frac{m}{\sqrt{T}} \ln T \ln \ln T \rightarrow 0$, it holds that $P\left\{a_{T} \Lambda_{T}^{\varepsilon *} \leq x+b_{T}\right\} \rightarrow$ $e^{-2 e^{-x}}$, where $a_{T}=\sqrt{2 \ln \ln T}$ and $b_{T}=2 \ln \ln T+\frac{p}{2} \ln \ln \ln T-\ln \Gamma\left(\frac{p}{2}\right)$, with $\Gamma(\cdot)$ the Gamma function. Under the alternative hypothesis defined in equation (18) in the main paper for $\Sigma_{\varepsilon}$, if equation (19) in the main paper holds, then $P\left[\Lambda_{T}^{\varepsilon *}>c_{\alpha, T}\right]=1$.

\section{Remarks}

T5.1 The main feature of Theorem 1 is that the same theory applies to residual-based tests as to observable data. This is due to the fact that, under our assumptions, an almost sure rate of convergence can be derived for the OLS estimator $\hat{\beta}$, with $\hat{\beta}-\beta=O_{a . s .}\left(\sqrt{\frac{\ln \ln T}{T}}\right)$. By virtue of this result, a SIP and a SLLN can be shown for the partial sums of the squared 
residuals, so that the asymptotics of tests based on $\Lambda_{T}^{\varepsilon}(\tau)$ can be derived in the same way as for tests based on $\Lambda_{T}(\tau)$.

T5.2 Theorem 1 can be readily extended to accommodate for the presence of linear or polynomial trends among the $x_{t}$ s; the only thing that changes is the rate of convergence of $\hat{\beta}$, which in presence of linear trends is actually faster than $O_{a . s .}\left(\sqrt{\frac{\ln \ln T}{T}}\right)$. A consequence of Theorem 1 is that the test developed in the main paper can be applied to data with nonzero mean, trends or even time dummies: it suffices to filter out such deterministics, and then apply the test to the resulting residuals.

\section{A VAR model for exchange rates: the stability of the covari- ance matrix of the error term}

In this section, we apply the test developed in Section 1 to a VAR, checking whether the covariance matrix of the error term is stable over time. Inspired by Carriero et al. (2009), we consider a VAR consisting of 4 exchange rates vis-a-vis the US Dollar. Specifically, we consider monthly averages of the following currencies: Euro, British Pound, Yen, Canadian Dollar; data are collected from January 1999 to April 2013, which entails a sample size (modulo the first entry) of $T=172$.

Two tests are carried out in parallel. We verify whether the covariance matrix of the error term as a whole is subject to any changes. Furthermore, we also test for the stability of each of the eigenvalues of the covariance matrix. Since we find very weak evidence of breaks (if any) in the whole matrix and in its spectrum, we do not carry out any analysis on the eigenvectors: the key finding in this section is that, despite a major event such as the 2007-2009 recession, the covariance matrix of the VAR model of exchange rates does not change over time.

Carriero et al. (2009) propose a Bayesian approach to model the exchange rates directly, imposing a prior on the VAR coefficients in order to shrink them towards a unit root representation. Preliminary analysis shows that, as expected, our series have a unit root. We therefore fit a VAR to the returns of the exchange rates. We report descriptive statistics for the returns, and a plot of the original series, in Table E1 and Figure A respectively.

\section{[Insert Table E1 and Figure A somewhere here]}

As the evidence in Carriero et al. (2009) shows, applying VAR models to exchange rates could yield a good level of forecasting accuracy, also in light of the fact that exchange rates have a tendency to co-move. However, Castle et al. (2010) find that when the covariance matrix of the error term in a regression changes, this could have pernicious effects on the forecasting ability of the model; thus, applying our test as discussed in Section 1 to the covariance matrix 
of the error term (and to its eigenvalues) could shed some light as to the forecasting ability of the VAR. Estimation results and mis-specification tests are reported in Table E2: the best specification was found to be a VAR of order 5, using lags 1,2 and 5. As can be seen from the Table, the model is correctly specified, save for the lack of normality in the error term; in view of Assumption R1, this is not an issue when applying our test. We also note that, as can be expected as regards the residuals of a correctly specified model, the error term is found to be serially uncorrelated, and we therefore estimate the long run variance without adjusting for serial correlation.

\section{[Insert Table E2 somewhere here]}

The test is applied, preliminarily, to the (lower triangular part of the) whole covariance matrix: the results in the Monte Carlo exercise suggest that the dimension of the matrix $(n=4$ in our context) affords the test to have the correct size and power versus the alternative of one or more breaks. Given that we consider only the lower triangular part of the matrix, this entails applying our test with $p=10$ constraints under the null, as opposed to $p=16$ constraints which is the case in the Monte Carlo exercise.

We also verify the stability of each of the four eigenvalues of the matrix. As in Section the Monte Carlo exercise, this procedure is implemented by controlling for the family-wise rejection rate though a Bonferroni correction. Critical values for individual tests of levels $\alpha_{I}=1 \%, 5 \%$ and $10 \%$, and for procedure level $\alpha_{P}=1 \%, 5 \%$ and $10 \%$, with the Bonferroni correction $\alpha_{I}=\frac{\alpha_{P}}{4}$ are in Table D.

Results are reported in Table E3.

\section{[Insert Table E3 somewhere here]}

As can be seen from the first panel of the Table, the matrix, as a whole, is stable over time: as pointed out above, despite the presence of a major event such as the 2007-2009 recession, the covariance structure of the error term of the VAR does not change. This could be interpreted in light of the stylised fact that exchange rates tend to co-move (see the discussion in Carriero et al., 2009). This finding is also reinforced by the second panel of Table E3, where we find that the first three largest eigenvalues (denoted as $\lambda_{1}-\lambda_{3}$ ) do not change over time. As far as the smallest one is concerned $\left(\lambda_{4}\right)$, some evidence of a change is indeed found. However, the test rejects the null of a stable eigenvalue only when applied to each eigenvalue separately. Conversely, when adjusting for the family-wise error, no break is found at $5 \%$ level (the null of no break is rejected at $10 \%$ level for $\lambda_{4}$ ); as mentioned in Section 4 in the main paper, this is a consequence of the Bonferroni correction yielding relatively conservative results. However, this suggests very weak evidence of a break in the smallest eigenvalue; the estimated breakdate is found to be around June 2008, which has the natural interpretation of being a consequence of the latest recession. 


\section{Data description}

The data for the first exercise (time stability of the principal components of the term structure) have been obtained from Datastream (source: ICAP), and they refer to the zero-coupon yield curve obtained from Treasury bond yields. We have employed the following tickers: US00Y01; US00Y03; US00Y06; US00Y09; US01Y00; US01Y03; US01Y06; US01Y09; US02Y00; US02Y06; US03Y00; US04Y00; US05Y00; US06Y00; US07Y00; US08Y00; US09Y00; US10Y00.

The data for the second exercise (time stability of the covariance matrix of a VAR fitted to major currencies) has been obtained from the FRED archive of the Federal Reserve Bank of St Louis, https://research.stlouisfed.org/fred2/release?rid=15.

\section{Useful lemmas and proofs}

In this section, we report the statements and proofs of Lemmas 1-4, frequently cited in the paper. We also report the necessary Lemmas for the results in Section 5, and the proof of Theorem 1.

We note that many of the result in this section are extensions of existing results in the literature - indeed, most of the results derived here are in Davidson (1994). Most results in Davidson (1994), however, are reported for the $L_{1}$-norm, whilst, when proving the SIP, we need essentially the same results but for the $L_{1+\epsilon}$-norm.

Lemma 1 Let $B$ and $\rho$ be non-negative random variables, with $|B|_{q^{*} /(q-1)}<\infty$ and $|\rho|_{q^{*}}<\infty$, where $q^{*}=q\left(1+\frac{\delta}{2}\right), q \geq 1$ and $\delta>0$. Assume $|B \rho|_{r}<\infty$ for $r>2+\delta$. Then

$$
\begin{aligned}
|B \rho|_{2+\delta} \leq & {\left[|\rho|_{q^{*}}|B|_{q^{*} /(q-1)}\right]^{\frac{r-(2+\delta)}{2 r-(2+\delta)}} \times } \\
& {\left[|B \rho|_{r}^{\frac{r}{2+\delta}-\frac{r(2+\delta-r)}{(2+\delta)-r}}+|B \rho|_{r}^{\frac{(2+\delta) r}{2 r-(2+\delta)}}\right] . }
\end{aligned}
$$

Proof. The proof is fairly similar to Gallant and White (1988; see Davidson, 1994, p. $271)$, who prove it for the $L_{2}$-norm. Let $C=\left[|\rho|_{q^{*}}|B|_{q^{*} /(q-1)}|B \rho|_{r}^{-r}\right]^{\frac{2+\delta}{2+\delta-2 r}}$, and define $B_{1}=$ $I_{\{B \rho \leq C\}} B$. By construction, $|B \rho|_{2+\delta} \leq\left|B_{1} \rho\right|_{2+\delta}+\left|\left(B-B_{1}\right) \rho\right|_{2+\delta}$. We have

$$
\begin{aligned}
\left|B_{1} \rho\right|_{2+\delta} & =\left[\int_{B \rho \leq C}(B \rho)^{2+\delta} d P\right]^{\frac{1}{2+\delta}} \\
& \leq C^{1 / 2}\left[\int_{B \rho \leq C}(B \rho)^{\frac{2+\delta}{2}} d P\right]^{\frac{2}{2+\delta} \frac{1}{2}} \\
& \leq C^{1 / 2}\left[|\rho|_{q^{*}}|B|_{q^{*} /(q-1)}\right]^{1 / 2},
\end{aligned}
$$

where the last passage follows from Holder's inequality. Also, since $r>2$ and, in this case, 
$(B \rho)^{r}>C^{r}$,

$$
\begin{aligned}
\left|\left(B-B_{1}\right) \rho\right|_{2+\delta} & =\left[\int_{B \rho>C}(B \rho)^{2+\delta} d P\right]^{\frac{1}{2+\delta}} \\
& \leq C^{1-\frac{r}{2+\delta}}\left[\int_{B \rho>C}(B \rho)^{r} d P\right]^{\frac{r}{2+\delta} \frac{1}{r}} \\
& \leq C^{1-\frac{r}{2+\delta}}|B \rho|_{r}^{\frac{r}{2+\delta}}
\end{aligned}
$$

Substituting for $C$ gives (2).

Lemma 2 Under Assumption 1, $w_{t}$ is $L_{2+\epsilon}-N E D$ of size $\alpha^{\prime}>\frac{1}{2}$ on $\left\{v_{t}\right\}_{t=-\infty}^{+\infty}$.

Proof. The proof follows similar passages as in Example 17.17 in Davidson (1994, p. 273); for simplicity, assume $n=1$. Let $x_{t}^{a}=w_{t}$ and $x_{t}^{b}=E\left[w_{t} \mid w_{t-m}, \ldots, w_{t+m}\right]$; and define, in a similar fashion, $y_{t}^{a}=y_{t}$ and $y_{t}^{b}=E\left[y_{t} \mid y_{t-m}, \ldots, y_{t+m}\right]$. Then

$$
\begin{aligned}
\left|x_{t}^{a}-x_{t}^{b}\right|_{2+\epsilon} & \leq\left|\left(\left|y_{t}^{a}\right|+\left|y_{t}^{b}\right|\right)\left(\left|y_{t}^{a}-y_{t}^{b}\right|\right)\right|_{2+\epsilon} \\
& =\left|B\left(y_{t}^{a}, y_{t}^{b}\right) \rho\left(y_{t}^{a}, y_{t}^{b}\right)\right|_{2+\epsilon}=|B \rho|_{2+\epsilon} .
\end{aligned}
$$

We begin with considering some preliminary results. First, we show that $|B \rho|_{r}<\infty$, uniformly in $m$. We have $|B \rho|_{r}<|B|_{2 r}|\rho|_{2 r}$, with $|B|_{2 r} \leq\left|y_{t}^{a}\right|_{2 r}+\left|y_{t}^{b}\right|_{2 r} \leq 2\left|y_{t}^{a}\right|_{2 r}$, having used Minkowski's inequality and conditional modulus inequalities; this is bounded due to Assumption 1(i), and the bound holds uniformly in $m$. Also, $|\rho|_{2 r} \leq 2\left|y_{t}^{a}\right|_{2 r}$ as a consequence of equation (17.4) in Davidson (1994, p. 262), again uniformly in $m$. Further, it holds that $|\rho|_{q^{*}}<\infty$ for $q^{*} \leq 2 r$, and thus for $q<2 r$; also, $|B|_{q^{*} /(q-1)}<\infty$ if $q^{*} \geq \frac{4}{3}$, i.e. $q>\frac{4}{3}$. Finally, note that for $q^{*} \leq 2$, $|\rho|_{q^{*}} \leq|\rho|_{2+\epsilon}$; by Assumption 1(i), $|\rho|_{2+\epsilon} \leq M_{t} v_{m}$, where $M_{t}$ can be set so that $M_{t} \leq 2 E\left\|y_{t}\right\|^{2+\epsilon}$, and $v_{m}$ is a non-negative sequence of size $v_{m}=O\left(m^{-\alpha}\right)$. Putting all these results together, and using Lemma 1, we have $\left|x_{t}^{a}-x_{t}^{b}\right|_{2+\epsilon}=\left|x_{t}^{a}-E\left[w_{t} \mid w_{t-m}, \ldots, w_{t+m}\right]\right|_{2+\epsilon} \leq M_{t}^{\prime} v_{m}^{\frac{r-(2+\epsilon)}{2 r-(2+\epsilon)}}=M_{t}^{\prime} v_{m}^{\prime}$; note that $M_{t}^{\prime}=M_{t}\left[|B \rho|_{r}^{\frac{r}{2+\epsilon}-\frac{r(2+\epsilon-r)}{(2+\epsilon)-r}}+|B \rho|_{r}^{\frac{(2+\epsilon) r}{2 r-(2+\epsilon)}}\right]|B|_{q^{*} /(q-1)}^{\frac{r-(2+\epsilon)}{2 r-(2+\epsilon)}}$ is a sequence of positive, finite numbers. The size of $v_{m}^{\prime}$ is $v_{m}^{\prime}=O\left(m^{-\alpha \frac{r-(2+\epsilon)}{2 r-(2+\epsilon)}}\right)=O\left(m^{-\alpha^{\prime}}\right)$. Assumption 1(ii) entails that $\alpha^{\prime}>\frac{1}{2}$.

Lemma 3 Under Assumption 1, vec $\left[\bar{w}_{t} \bar{w}_{t-l}^{\prime}-E\left(\bar{w}_{t} \bar{w}_{t-l}^{\prime}\right)\right]$ is $L_{1+\epsilon / 2}-N E D$ of size $\alpha^{\prime}$ on $\left\{v_{t}\right\}_{t=-\infty}^{+\infty}$, for every $l$.

Proof. The Lemma is an application of Theorem 17.9 in Davidson (1994, p. 268), where $L_{1^{-}}$and $L_{2}$-norms are replaced, respectively, by $L_{1+\epsilon / 2^{-}}$and $L_{2+\epsilon^{-} \text {-norms. }}$

Lemma 4 Under Assumption 1 and $2(i)(b)-(i i)$, vec $\left[\bar{w}_{t} \bar{w}_{t}^{\prime}-E\left(\bar{w}_{t} \bar{w}_{t}^{\prime}\right)\right]$ is $L_{2+\epsilon}-N E D$ of size $\alpha^{\prime}$ on $\left\{v_{t}\right\}_{t=-\infty}^{+\infty}$. 
Proof. The proof is very similar to that of Lemma 2. Assuming $n=1$ and letting $\omega_{t}=w_{t}^{2}$

$$
\begin{aligned}
\left|\omega_{t}^{a}-\omega_{t}^{b}\right|_{2+\epsilon} & \leq\left|\left(x_{t}^{a}+x_{t}^{b}\right)\left(x_{t}^{a}-x_{t}^{b}\right)\right|_{2+\epsilon} \\
& =\left|\left(\left|y_{t}^{a}\right|^{3}+\left|y_{t}^{b}\right|^{3}+\left|y_{t}^{a}\right|^{2}\left|y_{t}^{b}\right|+\left|y_{t}^{a}\right|\left|y_{t}^{b}\right|^{2}\right)\left(\left|y_{t}^{a}-y_{t}^{b}\right|\right)\right|_{2+\epsilon} \\
& =\left|B\left(y_{t}^{a}, y_{t}^{b}\right) \rho\left(y_{t}^{a}, y_{t}^{b}\right)\right|_{2+\epsilon}=|B \rho|_{2+\epsilon},
\end{aligned}
$$

so that the Lemma follows from Assumption 2(ii) and Lemma 1.

Lemma 5 Under Assumption R1, it holds that:

(i) $\bar{w}_{t}^{\varepsilon}$ is $L_{2+\epsilon}-N E D$ of size $\alpha^{\prime}>\frac{1}{2}$ on $\left\{v_{t}\right\}_{t=-\infty}^{+\infty}$;

(ii) redefining $\bar{w}_{t}^{\varepsilon}$ in a richer probability space, $\sum_{t=1}^{\lfloor T \tau\rfloor} \bar{w}_{t}^{\varepsilon}=\sum_{t=1}^{\lfloor T \tau\rfloor} X_{t}^{\varepsilon}+O_{a . s .}\left(\lfloor T \tau\rfloor^{\frac{1}{2}-\delta}\right)$, uniformly in $\tau$, where $X_{t}^{\varepsilon}$ is a zero mean, i.i.d. Gaussian sequence with $E\left(X_{t}^{\varepsilon} X_{t}^{\varepsilon \prime}\right)=V^{\varepsilon}$ and $\delta>0$

(iii) $\hat{\beta}-\beta=O_{a . s .}\left(\sqrt{\frac{\ln \ln T}{T}}\right)$;

(iv) defining $\overline{\hat{w}}_{t}^{\varepsilon}=\hat{w}_{t}^{\varepsilon}-\operatorname{vec}\left(\Sigma_{\varepsilon}\right)$, it holds that $\sum_{t=1}^{\lfloor T \tau\rfloor} \overline{\hat{w}}_{t}^{\varepsilon}=\sum_{t=1}^{\lfloor T \tau\rfloor} X_{t}^{\varepsilon *}+O_{a . s .}\left(\lfloor T \tau\rfloor^{\frac{1}{2}-\delta}\right)$, uniformly in $\tau$, where $X_{t}^{\varepsilon *}$ is a zero mean, i.i.d. Gaussian sequence with $E\left(X_{t}^{\varepsilon *} X_{t}^{\varepsilon * \prime}\right)=V^{\varepsilon}$ and $\delta>0$;

(v) vec $\left[\bar{w}_{t}^{\varepsilon} \bar{w}_{t-l}^{\varepsilon \prime}-E\left(\bar{w}_{t}^{\varepsilon} \bar{w}_{t-l}^{\varepsilon \prime}\right)\right]$ is $L_{1+\epsilon / 2}-N E D$ of size $\alpha^{\prime}$ on $\left\{v_{t}\right\}_{t=-\infty}^{+\infty}$, for every $l$.

Further, under Assumptions R1(ii) and R2(i)-(ii), it holds that:

(vi) $\operatorname{vec}\left[\bar{w}_{t}^{\varepsilon} \bar{w}_{t}^{\varepsilon \prime}-E\left(\bar{w}_{t}^{\varepsilon} \bar{w}_{t}^{\varepsilon \prime}\right)\right]$ is $L_{2+\epsilon}-N E D$ of size $\alpha^{\prime}$ on $\left\{v_{t}\right\}_{t=-\infty}^{+\infty}$.

Proof. Parts (i) and (v) of the Lemma are the same as Lemmas 2 and 3 respectively. Part (ii) is the same as equation (2) in the main paper, and we refer to the proof of Theorem 1 in the main paper for details. Similarly, part (vi) of the Lemma is the same as Lemma 4.

As far as part (iii) is concerned, let $n=q=1$ for the sake of notational simplicity, and consider $\hat{\beta}-\beta=\left(\sum_{t=1}^{T} x_{t}^{2}\right)^{-1}\left(\sum_{t=1}^{T} x_{t} \varepsilon_{t}\right)$. We start from the denominator. By virtue of Assumption R1(i), Lemma 2 entails that $x_{t}^{2}$ is $L_{2+\epsilon}$-NED of size $\alpha^{\prime}>\frac{1}{2}$ on the strong mixing basis $\left\{v_{t}\right\}_{t=-\infty}^{+\infty}$. This result allows to use a SLLN, since the assumptions of Theorem 2.1 in Ling (2007) are satisfied; thus, $\sum_{t=1}^{T} x_{t}^{2}=O_{a . s .}(T)$. Consider now the numerator; Assumptions $\mathrm{R} 1$ (i)-(ii) entail that $x_{t} \varepsilon_{t}$ is $L_{2+\epsilon}$-NED of size $\alpha^{\prime}>\frac{1}{2}$ on $\left\{v_{t}\right\}_{t=-\infty}^{+\infty}$; this is a direct application of Theorem 17.17 in Davidson (1994, p. 273), and it follows the same lines as the proof of Lemma 2. Using this result and Assumption R1(iii), a SIP holds, based on the same passages as in the proof of Theorem 1 in the main paper, so that $\sum_{t=1}^{T} x_{t} \varepsilon_{t}=\sum_{t=1}^{T} N_{t}^{x \varepsilon}+O_{a . s .}\left(T^{\frac{1}{2}-\delta}\right)$ for some $\delta>0$, where $N_{t}^{x \varepsilon}$ is a sequence of i.i.d. zero mean, Gaussian random variables with 
covariance matrix $V^{x \varepsilon}$. Therefore, by the Law of the Iterated Logarithm (LIL), $\sum_{t=1}^{T} x_{t} \varepsilon_{t}=$ $O_{a . s .}(\sqrt{T \ln \ln T})$. Part (iii) follows by combining the two results.

Finally, we show part (iv). We can write $\sum_{t=1}^{\lfloor T \tau\rfloor} \overline{\hat{w}}_{t}^{\varepsilon}=\sum_{t=1}^{\lfloor T \tau\rfloor} \bar{w}_{t}^{\varepsilon}-2 v e c\left[(\hat{\beta}-\beta)\left(\sum_{t=1}^{\lfloor T \tau\rfloor} x_{t} \varepsilon_{t}^{\prime}\right)\right]$ $+\operatorname{vec}\left[(\hat{\beta}-\beta)\left(\sum_{t=1}^{\lfloor T \tau} x_{t} x_{t}^{\prime}\right)(\hat{\beta}-\beta)^{\prime}\right]=\sum_{t=1}^{\lfloor T \tau\rfloor} \bar{w}_{t}^{\varepsilon}+I+I I$. By virtue of the passages above, $I$ and $I I$ are both $O_{a . s .}(\ln \ln T)$. Part (iv) then follows from part (ii).

Lemma 6 Under Assumptions R1(ii)-(iv)(a) and R2, it holds that $\sup _{1 \leq\lfloor T \tau\rfloor \leq T}\left\|\tilde{V}_{\tau}^{\varepsilon}-V^{\varepsilon}\right\|=$ $O_{p}\left(\frac{1}{m}\right)+O_{p}\left(m \ln T \sqrt{\frac{\ln \ln T}{T}}\right)$.

Proof. Let, preliminarily, $\tilde{V}_{\tau}^{\varepsilon \varepsilon}=\left(\hat{\Psi}_{0,\lfloor T \tau\rfloor}^{\varepsilon \varepsilon}+\hat{\Psi}_{0,1-\lfloor T \tau\rfloor}^{\varepsilon \varepsilon}\right)+\sum_{l=1}^{m}\left(1-\frac{l}{m}\right)\left[\left(\hat{\Psi}_{l,\lfloor T \tau\rfloor}^{\varepsilon \varepsilon}+\hat{\Psi}_{l,\lfloor T \tau\rfloor}^{\varepsilon \varepsilon \prime}\right)+\right.$ $\left.\left(\hat{\Psi}_{l, 1-\lfloor T \tau\rfloor}^{\varepsilon \varepsilon}+\hat{\Psi}_{l, 1-\lfloor T \tau\rfloor}^{\varepsilon \varepsilon \prime}\right)\right]$, where $\hat{\Psi}_{l,\lfloor T \tau\rfloor}^{\varepsilon \varepsilon}=\frac{1}{T-(l+1)} \sum_{t=l+1}^{\lfloor T \tau\rfloor}\left[w_{t}^{\varepsilon}-\operatorname{vec}\left(\hat{\Sigma}_{\varepsilon}\right)\right]\left[w_{t-l}^{\varepsilon}-\operatorname{vec}\left(\hat{\Sigma}_{\varepsilon}\right)\right]^{\prime}$, and similarly for $\hat{\Psi}_{l, 1-\lfloor T \tau\rfloor}^{\varepsilon \varepsilon}$. We start by showing that $\sup _{1 \leq\lfloor T \tau\rfloor \leq T}\left\|\tilde{V}_{\tau}^{\varepsilon \varepsilon}-V^{\varepsilon}\right\|=O_{p}\left(\frac{1}{m}\right)+$ $O_{p}\left(\frac{m}{\sqrt{T}}\right)$; the proof is very similar to that of Theorem 2 in the main paper, and therefore only the most relevant passages are reported. Let $\hat{\Sigma}_{\varepsilon \varepsilon}=T^{-1} \sum_{t=1}^{T} \varepsilon_{t} \varepsilon_{t}^{\prime}$, and note that $\hat{\Sigma}_{\varepsilon}-\hat{\Sigma}_{\varepsilon \varepsilon}=(\hat{\beta}-\beta)$ $\left(T^{-1} \sum_{t=1}^{T} x_{t} x_{t}^{\prime}\right)(\hat{\beta}-\beta)^{\prime}-\left(T^{-1} \sum_{t=1}^{T} \varepsilon_{t} x_{t}^{\prime}\right)(\hat{\beta}-\beta)^{\prime}-(\hat{\beta}-\beta)\left(T^{-1} \sum_{t=1}^{T} x_{t} \varepsilon_{t}^{\prime}\right)=I+I I$ $+I I^{\prime}$. By Lemma 5 , we have $I=O_{\text {a.s. }}\left(\frac{\ln \ln T}{T}\right)$, and similarly $I I=O_{a . s .}\left(\frac{\ln \ln T}{T}\right)$. Thus, for every $l, \hat{\Psi}_{l,\lfloor T \tau\rfloor}^{\varepsilon \varepsilon}=[T-(l+1)]^{-1} \sum_{t=l+1}^{\lfloor T \tau\rfloor}\left[w_{t}^{\varepsilon}-\operatorname{vec}\left(\hat{\Sigma}_{\varepsilon \varepsilon}\right)\right]\left[w_{t-l}^{\varepsilon}-v e c\left(\hat{\Sigma}_{\varepsilon \varepsilon}\right)\right]^{\prime}+O_{a . s .}\left(\frac{\ln \ln T}{T}\right)$. By using part (ii) of Lemma 5 , it follows that $\hat{\Sigma}_{\varepsilon \varepsilon}-\Sigma_{\varepsilon}=O_{a . s .}\left(T^{-\frac{1}{2}-\delta}\right)$, so that $\hat{\Psi}_{l,\lfloor T \tau\rfloor}^{\varepsilon \varepsilon}$ $=[T-(l+1)]^{-1} \sum_{t=l+1}^{\lfloor T \tau\rfloor} \bar{w}_{t}^{\varepsilon} \bar{w}_{t-l}^{\varepsilon \prime}+O_{a . s .}\left(T^{-\frac{1}{2}-\delta}\right)$; the same holds for $\hat{\Psi}_{l, 1-\lfloor T \tau\rfloor}^{\varepsilon \varepsilon}$. Therefore, $\hat{\Psi}_{l,\lfloor T \tau\rfloor}^{\varepsilon \varepsilon}+\hat{\Psi}_{l, 1-\lfloor T \tau\rfloor}^{\varepsilon \varepsilon}-\Psi_{l}^{\varepsilon \varepsilon}=[T-(l+1)]^{-1} \sum_{t=l+1}^{T}\left[\bar{w}_{t}^{\varepsilon} \bar{w}_{t-l}^{\varepsilon \prime}-E\left(\bar{w}_{t}^{\varepsilon} \bar{w}_{t-l}^{\varepsilon \prime}\right)\right]+O_{a . s .}\left(T^{-\frac{1}{2}-\delta}\right)$. Henceforth, the proof is the same as for equation (6) in the main paper, by virtue of part (vi) of Lemma 5 and since the assumptions on the autocovariances $\Psi_{l}^{\varepsilon}$ are the same as in Assumption 2. Finally, we show that $\sup _{1 \leq\lfloor T \tau\rfloor \leq T}\left\|\tilde{V}_{\tau}^{\varepsilon \varepsilon}-\tilde{V}_{\tau}^{\varepsilon}\right\|=O_{p}\left(m \sqrt{\frac{\ln \ln T}{T}}\right)$. Consider the quantities $I=[T-(l+1)]^{-1} \sum_{t=l+1}^{\lfloor T \tau\rfloor} 2 v e c\left[(\hat{\beta}-\beta)^{\prime} x_{t} \varepsilon_{t}^{\prime}\right]\left[w_{t-l}^{\varepsilon}-\operatorname{vec}\left(\hat{\Sigma}_{\varepsilon}\right)\right]^{\prime}$ and $I I=[T-(l+1)]^{-1} \sum_{t=l+1}^{\lfloor T \tau\rfloor} \operatorname{vec}\left[(\hat{\beta}-\beta)\left(x_{t} x_{t}^{\prime}\right)(\hat{\beta}-\beta)^{\prime}\right]\left[w_{t-l}^{\varepsilon}-\operatorname{vec}\left(\hat{\Sigma}_{\varepsilon}\right)\right]^{\prime}$. We have $I \leq$ $\|\hat{\beta}-\beta\|\left\{[T-(l+1)]^{-1} \sum_{t=l+1}^{\lfloor T \tau\rfloor}\left\|x_{t} \varepsilon_{t}^{\prime}\right\|^{2}\right\}^{1 / 2}\left\{[T-(l+1)]^{-1} \sum_{t=l+1}^{\lfloor T \tau\rfloor}\left\|w_{t-l}^{\varepsilon}-\operatorname{vec}\left(\hat{\Sigma}_{\varepsilon}\right)\right\|^{2}\right\}^{1 / 2}$. By applying Theorem 17.9 in Davidson (1994, p. 268) following similar lines as in the proof of Lemma $3,\left\|x_{t} \varepsilon_{t}^{\prime}\right\|^{2}$ can be shown to be $L_{1+\epsilon / 2}$ NED of size $\alpha^{\prime}>\frac{1}{2}$ on $\left\{v_{t}\right\}_{t=-\infty}^{+\infty}$; thus, a SLLN (see e.g. Theorem 2.1 in Ling, 2007) can be applied with $[\lfloor T \tau\rfloor-(l+1)]^{-1} \sum_{t=l+1}^{\lfloor T \tau\rfloor}\left\|x_{t} \varepsilon_{t}^{\prime}\right\|^{2}=$ $O_{\text {a.s. }}$ (1); similarly, part (v) of Lemma 5 entails a SLLN whereby $[\lfloor T \tau\rfloor-(l+1)]^{-1} \sum_{t=l+1}^{\lfloor T \tau\rfloor} \| w_{t-l}^{\varepsilon}$ $-\operatorname{vec}\left(\hat{\Sigma}_{\varepsilon}\right) \|^{2}=O_{\text {a.s. }}(1)$; hence, $I=O_{\text {a.s. }}\left(\sqrt{\frac{\ln \ln T}{T}}\right)$. By the same logic, $I I=O_{\text {a.s. }}\left(\frac{\ln \ln T}{T}\right)$. After some algebra, $\sup _{1 \leq\lfloor T \tau\rfloor \leq T}\left\|\tilde{V}_{\tau}^{\varepsilon \varepsilon}-\tilde{V}_{\tau}^{\varepsilon}\right\|=O_{p}\left(m \sqrt{\frac{\ln \ln T}{T}}\right)$ follows. 
Lemma 7 Under Assumptions $R 1$ and R3, it holds that as $T \rightarrow \infty$, uniformly in $\tau, \hat{\lambda}_{i, \tau}^{\varepsilon}-\lambda_{i}^{\varepsilon}$ $=\left(x_{i}^{\varepsilon \prime} \otimes x_{i}^{\varepsilon \prime}\right) \operatorname{vec}\left(\hat{\Sigma}_{\varepsilon, \tau}-\Sigma_{\varepsilon}\right)+O_{p}\left(T^{-1}\right), \hat{x}_{i, \tau}^{\varepsilon}-x \varepsilon_{i}=v_{x, i}^{\varepsilon} \operatorname{vec}\left(\hat{\Sigma}_{\varepsilon, \tau}-\Sigma_{\varepsilon}\right)+O_{p}\left(T^{-1}\right)$ and $\hat{\gamma}_{i, \tau}^{\varepsilon}-\gamma_{i}^{\varepsilon}=v_{\gamma, i}^{\varepsilon} \operatorname{vec}\left(\hat{\Sigma}_{\varepsilon, \tau}-\Sigma_{\varepsilon}\right)+O_{p}\left(T^{-1}\right)$.

Proof. By part (i) of Lemma 5, an FCLT holds for vec $\left(\hat{\Sigma}_{\varepsilon, \tau}-\Sigma_{\varepsilon}\right)$, so that $\sup _{\lfloor T \tau\rfloor}\left\|\hat{\Sigma}_{\varepsilon, \tau}-\Sigma_{\varepsilon}\right\|$ $=O_{p}\left(T^{-1 / 2}\right)$ - see the proof of equation (1) in Theorem 1 in the main paper. Henceforth, the proof is the same as for Proposition 1 in the main paper.

Proof of Theorem 1. By Lemma 6, it suffices to show that $\mid \sup _{\frac{1}{T} \leq \tau \leq 1-\frac{1}{T}} \AA_{i T}^{\varepsilon}(\tau)-$ $\sup _{\frac{1}{T} \leq \tau \leq 1-\frac{1}{T}} M^{\varepsilon}(\tau) \mid=o_{p}\left[(\ln \ln T)^{-1 / 2}\right]$, where $M^{\varepsilon}(\tau)=\left[\sum_{i=1}^{p} \frac{B_{1 i}^{2}(\tau)}{\tau(1-\tau)}\right]^{1 / 2}, B_{1 i}(\tau)$ is a sequence of standard, independent Brownian bridges for $i=1, \ldots, p$, and

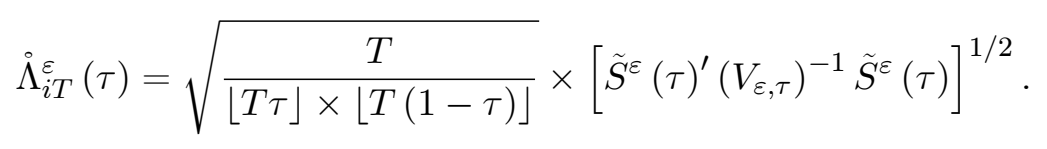

Indeed, we can show that $\left|\sup _{\frac{1}{T} \leq \tau \leq 1-\frac{1}{T}} \ddot{\Lambda}_{i T}^{\varepsilon}(\tau)-\sup _{\frac{1}{T} \leq \tau \leq 1-\frac{1}{T}} \stackrel{\complement}{i T}_{i T}^{\varepsilon}(\tau)\right|=O_{p}\left(\frac{\ln \ln T}{T}\right)$, where

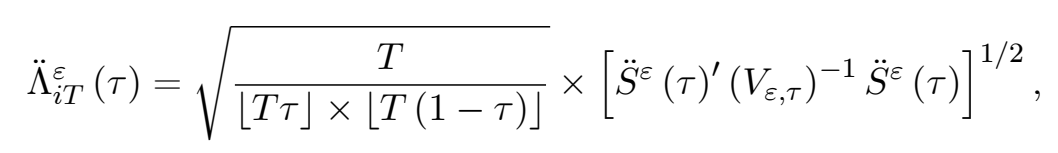

and $\ddot{S}^{\varepsilon}(\tau)=\sum_{t=1}^{\lfloor T \tau\rfloor} \operatorname{vec}\left(\varepsilon_{t} \varepsilon_{t}^{\prime}\right)$ with $\ddot{S}^{\varepsilon}(\tau)=0$ for $\tau \leq \frac{1}{T}$ or $\geq 1-\frac{1}{T}$. Since $\mid \sup _{\frac{1}{T} \leq \tau \leq 1-\frac{1}{T}} \ddot{\Lambda}_{i T}^{\varepsilon}(\tau)$ $-\sup _{\frac{1}{T} \leq \tau \leq 1-\frac{1}{T}} \AA_{i T}^{\varepsilon}(\tau)\left|\leq \sup _{\frac{1}{T} \leq \tau \leq 1-\frac{1}{T}}\right| \ddot{\Lambda}_{i T}^{\varepsilon}(\tau)-\AA_{i T}^{\varepsilon}(\tau) \mid$, the passages in the proof of Lemma 6 and the same logic as in the proof of Theorem 2 in the main paper yield $\sup _{\frac{1}{T} \leq \tau \leq 1-\frac{1}{T}} \mid \ddot{\Lambda}_{i T}^{\varepsilon}(\tau)-$ $\AA_{i T}^{\varepsilon}(\tau) \mid=O_{p}\left(\frac{\ln \ln T}{T}\right)$. Thus, it suffices to show that $\left|\sup _{\frac{1}{T} \leq \tau \leq 1-\frac{1}{T}} \ddot{\Lambda}_{i T}^{\varepsilon}(\tau)-\sup _{\frac{1}{T} \leq \tau \leq 1-\frac{1}{T}} M^{\varepsilon}(\tau)\right|$ $=o_{p}\left[(\ln \ln T)^{-1 / 2}\right]$. This can be shown following the passages in the proof of Theorem 3 in the main paper, using the SIP in part (ii) of Lemma 5 and Lemma 7. The behaviour of the test statistic under the alternative can be shown following the same lines as the proof of Theorem 4 in the main paper. 


\section{Tables}

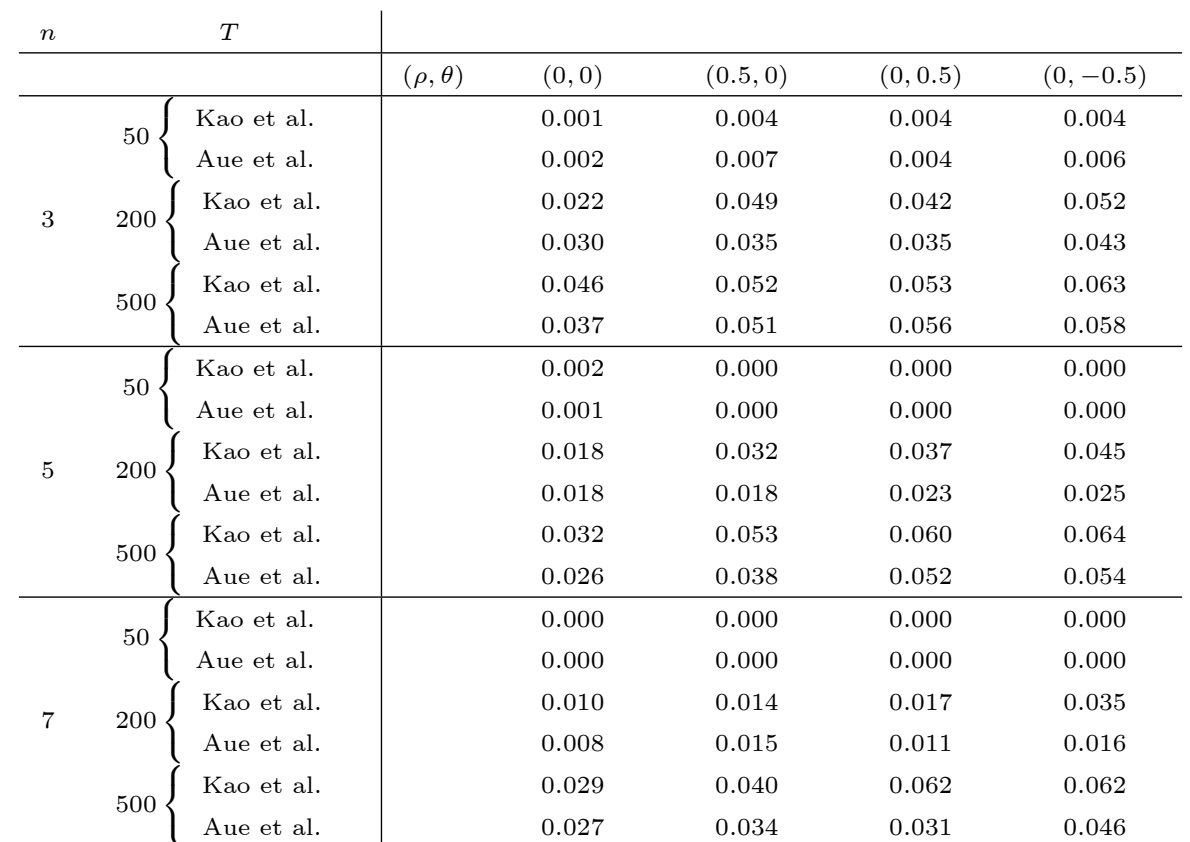

Table A1. Empirical rejection frequencies for the null of no change in $\Sigma$, based on a test for the stability of all eigenvalues. Data are generated according to equation (22) in the main paper. 


\begin{tabular}{|c|c|c|c|c|c|c|c|c|}
\hline \multirow[t]{2}{*}{$n$} & \multirow{2}{*}{\multicolumn{2}{|c|}{$T$}} & \multicolumn{5}{|c|}{$\Delta=\sqrt{\frac{\ln \ln T}{T^{1 / 2}}}$} & \multirow[t]{2}{*}{ first element only } \\
\hline & & & $(\rho, \theta)$ & $(0,0)$ & $(0.5,0)$ & $(0,0.5)$ & $(0,-0.5)$ & \\
\hline \multirow{6}{*}{3} & \multirow{2}{*}{50\{} & Kao et al. & & 0.027 & 0.034 & 0.037 & 0.034 & 0.077 \\
\hline & & Aue et al. & & 0.176 & 0.155 & 0.138 & 0.145 & 0.128 \\
\hline & \multirow{2}{*}{200} & Kao et al. & & 0.834 & 0.740 & 0.716 & 0.670 & 0.903 \\
\hline & & Aue et al. & & 0.968 & 0.902 & 0.879 & 0.854 & 0.966 \\
\hline & \multirow{2}{*}{500} & Kao et al. & & 0.998 & 0.987 & 0.985 & 0.961 & 0.990 \\
\hline & & Aue et al. & & 1.000 & 1.000 & 1.000 & 1.000 & 1.000 \\
\hline \multirow{6}{*}{5} & \multirow{2}{*}{50\{} & Kao et al. & & 0.000 & 0.000 & 0.001 & 0.000 & 0.000 \\
\hline & & Aue et al. & & 0.032 & 0.026 & 0.029 & 0.027 & 0.044 \\
\hline & \multirow{2}{*}{200} & Kao et al. & & 0.969 & 0.884 & 0.875 & 0.832 & 0.805 \\
\hline & & Aue et al. & & 0.998 & 0.980 & 0.975 & 0.951 & 0.921 \\
\hline & \multirow{2}{*}{500} & Kao et al. & & 1.000 & 0.999 & 1.000 & 0.998 & 0.996 \\
\hline & & Aue et al. & & 1.000 & 1.000 & 1.000 & 0.999 & 1.000 \\
\hline \multirow{6}{*}{7} & \multirow{2}{*}{50\{} & Kao et al. & & 0.000 & 0.000 & 0.000 & 0.000 & 0.000 \\
\hline & & Aue et al. & & 0.000 & 0.000 & 0.000 & 0.000 & 0.007 \\
\hline & \multirow{2}{*}{200\{} & Kao et al. & & 0.973 & 0.922 & 0.905 & 0.878 & 0.699 \\
\hline & & Aue et al. & & 1.000 & 0.993 & 0.992 & 0.987 & 0.850 \\
\hline & \multirow{2}{*}{500\{} & Kao et al. & & 1.000 & 1.000 & 1.000 & 1.000 & 0.994 \\
\hline & & Aue et al. & & 1.000 & 1.000 & 1.000 & 1.000 & 1.000 \\
\hline
\end{tabular}

Table A2. Power of the test for the null of no changes in $\Sigma$, based on a test for the stability of all eigenvalues.

Data are generated according to equation (22) in the main paper and under the alternative hypothesis specified in equation (25) in the main paper. The last column refers to a break of the same size, but only for the first element of $\Sigma$. 


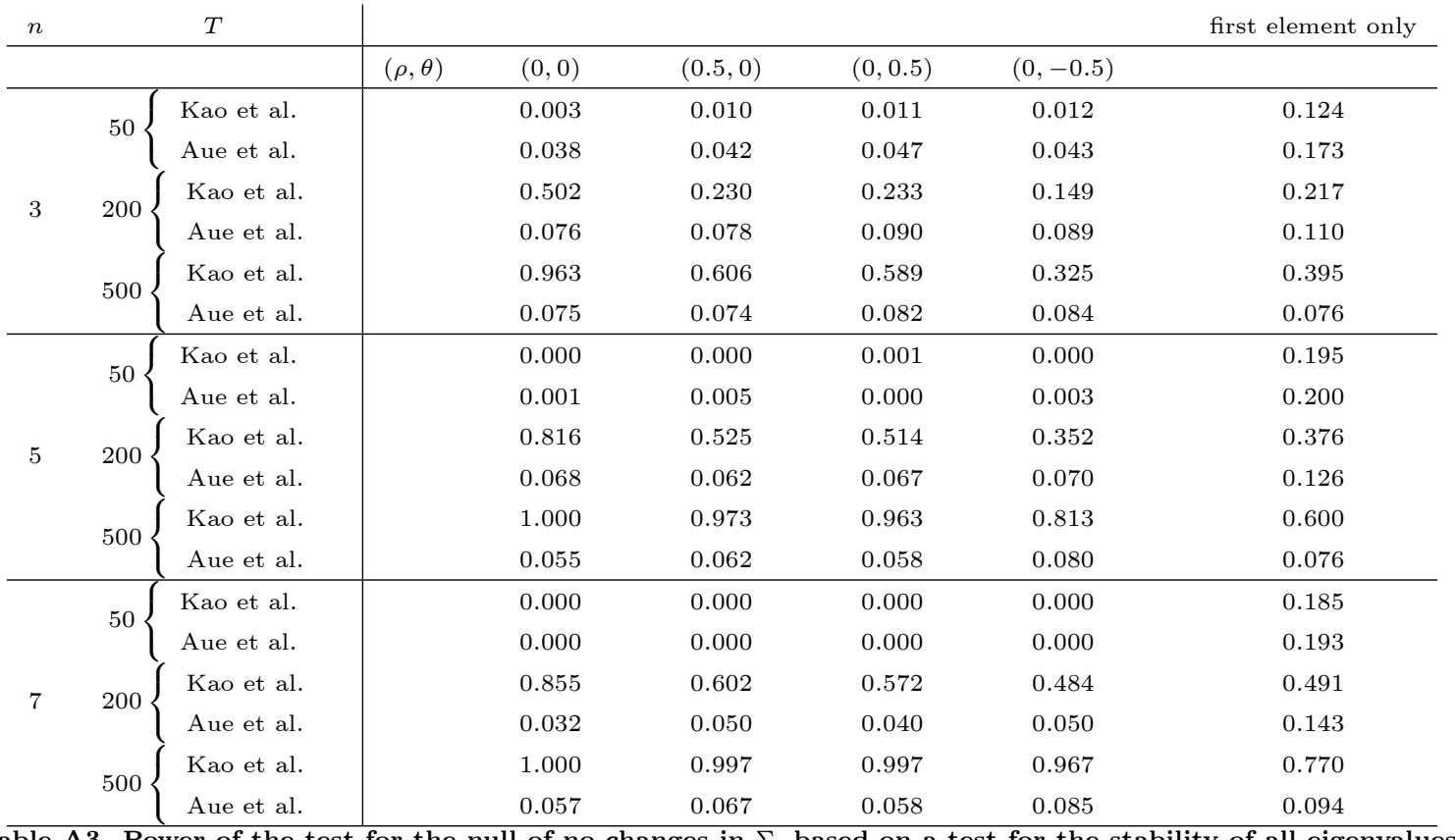

Table A3. Power of the test for the null of no changes in $\Sigma$, based on a test for the stability of all eigenvalues. Data are generated according to equation (22) in the main paper and under the alternative hypothesis specified in equation (27) in the main paper, with $k=T h_{n T}+1$ and $\Delta=I_{n}$. The last column refers to a case where only the first element of $\Sigma$ undergoes the break specified before. 


\begin{tabular}{|c|c|c|c|c|c|c|c|}
\hline$n$ & $T$ & size & power, mid-sample & power, non mid-sample & size & power, mid-sample & power, non mid-sample \\
\hline \multirow{4}{*}{3} & 50 & 0.053 & 0.240 & 0.356 & 0.050 & 0.309 & 0.568 \\
\hline & 100 & 0.046 & 0.395 & 0.347 & 0.035 & 0.471 & 0.776 \\
\hline & 200 & 0.065 & 0.773 & 0.469 & 0.063 & 0.838 & 0.993 \\
\hline & 500 & 0.061 & 0.941 & 0.651 & 0.050 & 0.958 & 1.000 \\
\hline \multirow{4}{*}{5} & 50 & 0.046 & 0.193 & 0.292 & 0.037 & 0.260 & 0.331 \\
\hline & 100 & 0.062 & 0.334 & 0.383 & 0.055 & 0.472 & 0.464 \\
\hline & 200 & 0.057 & 0.631 & 0.556 & 0.061 & 0.775 & 0.648 \\
\hline & 500 & 0.066 & 0.877 & 0.935 & 0.065 & 0.955 & 0.954 \\
\hline \multirow{4}{*}{7} & 50 & 0.041 & 0.180 & 0.204 & 0.046 & 0.274 & 0.261 \\
\hline & 100 & 0.053 & 0.284 & 0.237 & 0.050 & 0.457 & 0.263 \\
\hline & 200 & 0.062 & 0.628 & 0.268 & 0.067 & 0.739 & 0.325 \\
\hline & 500 & 0.059 & 0.844 & 0.518 & 0.065 & 0.945 & 0.632 \\
\hline
\end{tabular}

Table B1. Size and power versus a break in the first principal component. Data are simulated as indicated in the main paper; particularly, the first eigenvalue of the covariance matrix is set to 1 ; the others are simulated from a Uniform distribution with support [0,0.02], thereby having mean 0.01. The last three columns contain empirical rejection frequencies unde rthe same set-up, but all eigenvalues are of comparable magnitude, having mean 1. 


\begin{tabular}{|c|c|c|c|c|c|c|c|}
\hline$n$ & $T$ & size & power, mid-sample & power, non mid-sample & size & power, mid-sample & power, non mid-sample \\
\hline \multirow{4}{*}{3} & 50 & 0.048 & 0.308 & 0.386 & 0.041 & 0.190 & 0.056 \\
\hline & 100 & 0.053 & 0.559 & 0.673 & 0.050 & 0.431 & 0.106 \\
\hline & 200 & 0.043 & 0.862 & 0.915 & 0.064 & 0.759 & 0.233 \\
\hline & 500 & 0.055 & 0.982 & 0.999 & 0.067 & 0.917 & 0.510 \\
\hline \multirow{4}{*}{5} & 50 & 0.040 & 0.446 & 0.369 & 0.039 & 0.189 & 0.031 \\
\hline & 100 & 0.056 & 0.752 & 0.674 & 0.061 & 0.494 & 0.112 \\
\hline & 200 & 0.047 & 0.944 & 0.930 & 0.069 & 0.818 & 0.229 \\
\hline & 500 & 0.061 & 0.995 & 0.995 & 0.050 & 0.961 & 0.520 \\
\hline \multirow{4}{*}{7} & 50 & 0.048 & 0.479 & 0.386 & 0.040 & 0.210 & 0.049 \\
\hline & 100 & 0.045 & 0.768 & 0.654 & 0.063 & 0.489 & 0.122 \\
\hline & 200 & 0.053 & 0.949 & 0.918 & 0.064 & 0.816 & 0.221 \\
\hline & 500 & 0.061 & 0.996 & 1.000 & 0.060 & 0.964 & 0.497 \\
\hline
\end{tabular}

Table B2. Size and power for the test for the null of no changes in the largest eigenvalue of $\Sigma$, in presence of a large autoregressive root, after pre-whitening. Data are simulated as indicated in the main paper. 


\begin{tabular}{|c|c|c|c|c|c|c|c|c|c|c|c|c|}
\hline & \multicolumn{6}{|c|}{ monthly data } & \multicolumn{6}{|c|}{ weekly data } \\
\hline & mean & std. dev & skew & kurt & $\operatorname{AR}(1)$ & $\operatorname{ARCH}(7)$ & mean & std. dev & skew & kurt & $\operatorname{AR}(1)$ & $\operatorname{ARCH}(7)$ \\
\hline $1 m$ & -0.035 & 0.438 & -0.481 & 25.266 & -0.129 & $0.000 * * *$ & -0.007 & 0.159 & 0.119 & 66.052 & 0.151 & $0.000^{* * *}$ \\
\hline $3 m$ & -0.035 & 0.366 & -0.030 & 24.291 & 0.097 & $0.000 * * *$ & -0.008 & 0.121 & -0.664 & 46.650 & 0.324 & $0.000^{* * *}$ \\
\hline $6 m$ & -0.036 & 0.295 & -1.591 & 15.137 & 0.209 & $0.011^{* *}$ & -0.008 & 0.108 & -1.525 & 21.640 & 0.215 & $0.000^{* * *}$ \\
\hline $9 m$ & -0.037 & 0.274 & -1.624 & 10.921 & 0.257 & 0.668 & -0.008 & 0.121 & -1.301 & 14.454 & 0.035 & $0.000^{* * *}$ \\
\hline $12 m$ & -0.037 & 0.262 & -1.188 & 8.251 & 0.286 & 0.989 & -0.008 & 0.136 & -1.252 & 16.110 & -0.098 & $0.000^{* * *}$ \\
\hline $15 \mathrm{~m}$ & -0.038 & 0.267 & -0.851 & 6.835 & 0.268 & 0.972 & -0.008 & 0.130 & -0.883 & 9.984 & -0.049 & $0.000^{* * *}$ \\
\hline $18 m$ & -0.038 & 0.273 & -0.549 & 5.589 & 0.242 & 0.957 & -0.008 & 0.127 & -0.474 & 6.827 & -0.014 & $0.000^{* * *}$ \\
\hline $21 m$ & -0.038 & 0.282 & -0.323 & 4.695 & 0.210 & 0.962 & -0.008 & 0.129 & -0.138 & 5.693 & -0.003 & $0.000^{* * *}$ \\
\hline $24 m$ & -0.038 & 0.294 & -0.160 & 4.086 & 0.172 & 0.974 & -0.008 & 0.134 & 0.026 & 5.298 & -0.012 & $0.000^{* * *}$ \\
\hline $30 m$ & -0.038 & 0.303 & -0.043 & 3.937 & 0.146 & 0.984 & -0.008 & 0.139 & 0.080 & 4.930 & -0.022 & $0.000^{* * *}$ \\
\hline $3 y$ & -0.038 & 0.314 & 0.052 & 3.982 & 0.118 & 0.980 & -0.007 & 0.144 & 0.101 & 4.741 & -0.029 & $0.000^{* * *}$ \\
\hline $4 y$ & -0.036 & 0.319 & 0.060 & 4.198 & 0.073 & 0.970 & -0.007 & 0.149 & 0.041 & 4.560 & -0.045 & $0.000^{* * *}$ \\
\hline $5 y$ & -0.035 & 0.323 & 0.116 & 4.706 & 0.037 & 0.988 & -0.006 & 0.152 & 0.007 & 4.486 & -0.051 & $0.000^{* * *}$ \\
\hline $6 y$ & -0.033 & 0.321 & 0.140 & 5.151 & 0.033 & 0.994 & -0.006 & 0.152 & -0.029 & 4.555 & -0.052 & $0.000 * * *$ \\
\hline $7 y$ & -0.032 & 0.320 & 0.144 & 5.560 & 0.023 & 0.995 & -0.005 & 0.152 & -0.028 & 4.596 & -0.049 & $0.000^{* * *}$ \\
\hline $8 y$ & -0.031 & 0.318 & 0.138 & 5.868 & 0.017 & 0.994 & -0.005 & 0.152 & -0.033 & 4.776 & -0.044 & $0.000^{* * *}$ \\
\hline $9 y$ & -0.030 & 0.318 & 0.112 & 6.251 & 0.009 & 0.992 & -0.005 & 0.151 & -0.063 & 4.968 & -0.047 & $0.000^{* * *}$ \\
\hline $10 y$ & -0.030 & 0.318 & 0.048 & 6.653 & -0.001 & 0.990 & -0.005 & 0.151 & -0.059 & 4.946 & -0.046 & $0.000^{* * *}$ \\
\hline
\end{tabular}

Table C. Descriptive statistics for monthly and weekly data. We report the mean of the returns, the standard deviation, the skewness and the kurtosis, the AR(1) coefficient and the $p$-value of the $\operatorname{ARCH}(7)$ test for the returns - *,**, and $* * *$ denote rejection of the null of no ARCH effects at $10 \%, 5 \%$ and $1 \%$ levels respectively. 


\begin{tabular}{cc|ccc} 
& level & $10 \%$ & $5 \%$ & $1 \%$ \\
$(T, p)$ & & & & \\
\hline \multirow{3}{*}{$(163,1)$} & $c v_{1}$ & 2.8044 & 3.0716 & 3.5758 \\
& $c v_{3}$ & 3.1987 & 3.4222 & 3.9344 \\
& $c v_{18}$ & 3.7843 & 3.9690 & 4.1273 \\
\hline \multirow{3}{*}{$(712,1)$} & $c v_{1}$ & 2.9344 & 3.1861 & 3.6524 \\
& $c v_{3}$ & 3.3197 & 3.5131 & 3.9321 \\
& $c v_{18}$ & 3.8242 & 3.9655 & 4.3805 \\
\hline \multirow{3}{*}{$(163,18)$} & $c v_{1}$ & 6.1897 & 6.4075 & 6.7747 \\
\hline \multirow{2}{*}{$(712,18)$} & $c v_{3}$ & 6.5068 & 6.6486 & 7.0053 \\
\hline \multirow{3}{*}{$(166,1)$} & $c v_{3}$ & 6.2903 & 6.5016 & 6.9779 \\
\hline$(166,10)$ & $c v_{1}$ & 6.6279 & 6.8287 & 7.1674 \\
\hline & & 3.7952 & 3.0336 & 3.5951 \\
& & 5.0741 & 5.317 & 3.9874 \\
\hline
\end{tabular}

Table D. Critical values for the applications in Section 4 in the main paper, and in Section 2 in this supplement. In the Table, the notation $c v_{N}$ refers to the critical value to be used when $N$ hypotheses are being tested for, in order to have a procedure-wise level of $\mathbf{1 0 \%}, \mathbf{5 \%}$ and $\mathbf{1 \%}$ respectively. The panels with $(T, p)=(163,1)$ and $(732,1)$ contain critical values for unidimensional tests (monthly and weekly frequencies respectively), and therefore are used to test for changes in eigenvalues or when verifying the stability of the diagonal elements of $\Sigma$ one at a time. Panels where $(T, p)=(163,1)$ and $(732,1)$ contain critical values for tests with 18 hypotheses under the null, and thus are designed for tests for the stability of one eigenvector. Finally, panels with $(T, p)=(166,1)$ and $(166,10)$ contain critical

values for the application in Section 2: the former are for changes in the eigenvalues, both individual and family-wise tests unidimensional tests (monthly and weekly frequencies respectively); the latter are for testing the stability of the whole matrix. 


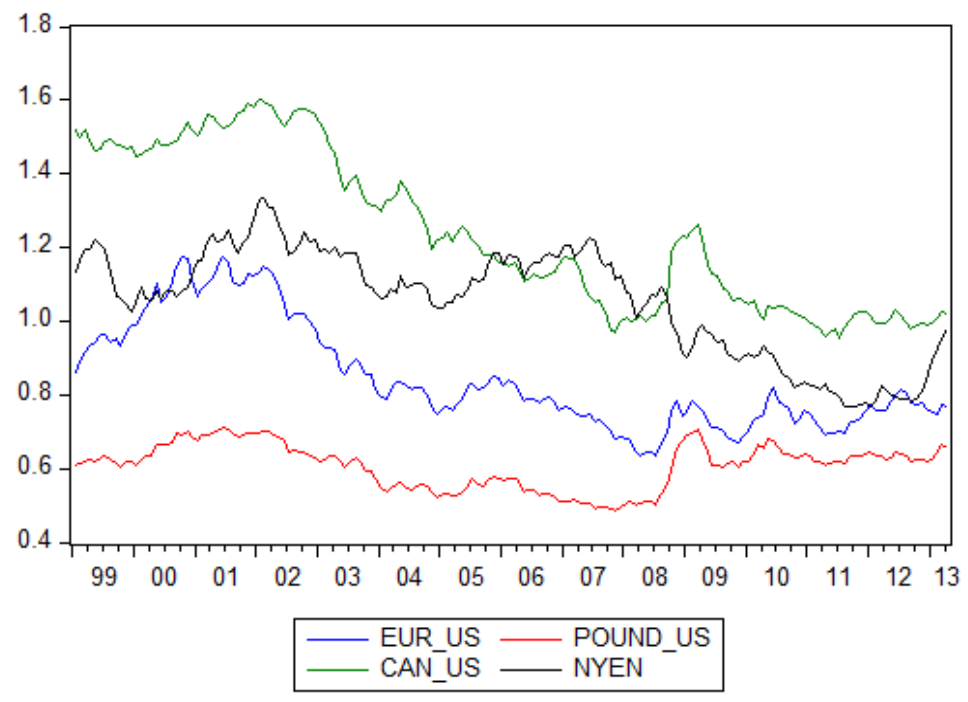

Figure A. Exchange rates vis-a-vis the US Dollar. Data are monthly, recorded between January 1999 and April 2013; as far as the Yen/US Dollar exchange rate is concerned, the original data have been divided by 100 to make figures comparable with the other exchange rates.

\begin{tabular}{c||cccccc} 
& & & & & & \\
& mean & std. dev & skew & kurt & $\operatorname{AR}(1)$ & $\operatorname{ARCH}(7)$ \\
\hline EUR & -0.0007 & 0.0252 & -0.0026 & 2.990 & 0.297 & 0.113 \\
GBP & 0.0004 & 0.0219 & 0.4460 & 4.841 & 0.294 & $0.000^{* * *}$ \\
CAN & -0.0023 & 0.0192 & 0.9417 & 9.774 & 0.272 & 0.505 \\
YEN & -0.0009 & 0.0238 & -0.1859 & 2.983 & 0.263 & 0.385
\end{tabular}

Table E1. Descriptive statistics for the returns on (vis-a-vis the US Dollar): the Euro (EUR), the British Pound (GBP), the Canadian Dollar (CAN) and the Yen (YEN). See Table 4 for an explanation of the output. 


\begin{tabular}{cl|cccc} 
& \multicolumn{5}{c}{ Estimation Output } \\
\cline { 3 - 4 } Constant & & EUR & GBP & CAN & YEN \\
\hline \multirow{2}{*}{ Lag 1 } & & & & \\
& EUR & -0.0015 & 0.0004 & -0.0020 & -0.0004 \\
& GBP & $0.3760^{*}$ & $0.2080^{*}$ & 0.1604 & $-0.0408^{*}$ \\
& CAN & -0.1642 & 0.0442 & -0.1053 & -0.0650 \\
& YEN & $0.2966^{*}$ & $0.4057^{*}$ & $0.2786^{*}$ & 0.0667 \\
& EUR & -0.0389 & -0.0139 & -0.0396 & 0.2045 \\
& GBP & $-0.2868^{*}$ & $-0.2044^{*}$ & -0.1499 & -0.0674 \\
& CAN & 0.2425 & $0.2680^{*}$ & 0.1300 & -0.0614 \\
& YEN & -0.1387 & -0.1891 & 0.0099 & 0.0169 \\
& EUR & 0.1462 & -0.0405 & 0.0394 & 0.1195 \\
& GBP & 0.0710 & 0.0165 & $0.2031^{*}$ & 0.0779 \\
& CAN & 0.0500 & -0.1051 & -0.0851 & -0.1171 \\
& YEN & -0.1385 & 0.0119 & -0.1180 & $0.2350^{*}$ \\
& & -0.1603 & -0.0684 & $-0.1311^{*}$ & $-0.2036^{*}$ \\
& & & & \\
\hline
\end{tabular}

\begin{tabular}{c|c}
\multicolumn{2}{c}{ Mis-specification tests } \\
\hline Serial Correlation LM test & 134.378 \\
& {$[0.904]$} \\
Normality & 100.308 \\
& {$[0.000]$} \\
\hline
\end{tabular}

Table E2. Estimation results and misspecification analysis for the VAR; the components of the vector are the following (vis-a-vis the US Dollar): the Euro (EUR), the British Pound (GBP), the Canadian Dollar (CAN) and the Yen (YEN). In the top half of the Table we report the estimation results: the symbol "*" denotes rejection of the null that the corresponding coefficient is zero at $\mathbf{5 \%}$ level. The second half of the Table contains misspecification tests: we report (in order of appearance) the portmanteu serial correlation test (up to lag 12) and the Jarque-Bera test. 


\begin{tabular}{|c|c|c|c|}
\hline \multirow{4}{*}{$H_{0}: \Sigma$ constant } & \multirow{4}{*}{4.8049} & $H_{0}: \lambda_{1}$ constant & 1.5457 \\
\hline & & $H_{0}: \lambda_{2}$ constant & 2.2527 \\
\hline & & $H_{0}: \lambda_{3}$ constant & 1.7077 \\
\hline & & $H_{0}: \lambda_{4}$ constant & $\begin{array}{c}3.4154^{*} \\
{[\text { June 2008] }}\end{array}$ \\
\hline
\end{tabular}

Table E3. Tests for changes in the covariance matrix of the error term of the VAR estimated in Table E2. Rejection at $10 \%, 5 \%$ and $1 \%$ levels are denoted with ${ }^{*},{ }^{* *}$ and ${ }^{* * *}$ respectively. Where present, numbers in square brackets are the estimated breakdates, defined as $T \times \arg \max \Lambda_{T}^{\varepsilon}(\tau)$. 


\section{References}

Aue, A., Horváth, L., Huskova, M., 2012. Segmenting mean-nonstationary time series via trending regressions. Journal of Econometrics 168, 367-381.

Bai, J., 2000. Vector autoregressive models with structural changes in regression coefficients and in variance-covariance matrices. Annals of Economics and Finance 1, 303-339.

Carriero, A., Kapetanios, G., Marcellino, M., 2009. Forecasting exchange rates with a large Bayesian VAR. International Journal of Forecasting 25, 400-417.

Castle, J.R., Fawcett, N., Hendry, D.F., 2010. Forecasting with equilibrium-correction models during structural breaks. Journal of Econometrics 158, 25-36.

Davidson, J., 1994. Stochastic Limit Theory. Oxford: Oxford University Press.

Gallant, A.R., White, H., 1988. A unified theory of estimation and inference for nonlinear dynamic models. Oxford: Basil Blackwell.

Ling, S., 2007. Testing for change points in time series models and limiting theorems for NED sequences. Annals of Statistics 35, 1213-1237. 